\title{
Recombinant lipid transfer protein Tri a 14: a novel heat and proteolytic resistant tool for the diagnosis of baker's asthma
}

\author{
A. Palacin ,J. Varela', S. Quirce ,V. del Pozo, L. Tordesillas , P. Barranco, M. Fernandez-Nieto ,J. Sastre ,A. Diaz-Perales \\ and G. Salcedo \\ Unidad de Bioquimica, Departamento de Biotecnología, E.TS. Ingenieros Agronomos, Madrid, Spain. Servicio de Ouimica de Proteinas, Centro de Investigaciones \\ Biológicas, CSIC, Madrid, Spain, Senvicio de Alergia, Hospital Universitario La Paz, Madrid, Spain, Servicio de Immunologia, fundacion Jimenez Diaz, Madrid, Spain \\ and" "Servicio de Alergia, Fundacion Jimenez Diaz, Madrid, Spain
}

\begin{abstract}
Summary
Background Baker's asthma is an important occupational allergic disease. Wheat lipid transfer protein (LTP) Tri a 14 is a major allergen associated with wheat allergy. No panel of wheat recombinant allergens for component-resolved diagnosis of baker's asthma is currently available.

Objective To evaluate the potential role of recombinant Tri a 14 as a novel tool for the diagnosis of baker's asthma, and to test the heat and proteolytic resistance of the wheat LTP allergen.

Methods A cDNA encoding Tri a 14 was isolated and sequenced, the recombinant allergen produced in Pichia pastoris and purified by chromatographic methods. Physicochemical and immunological comparison of the natural and recombinant forms of Tri a 14 was carried out by $\mathrm{N}$-terminal amino acid sequencing, matrix-assisted laser desorption/ionization mass spectrometry, circular dichroism (CD) analysis, IgE immunodetection, and specific IgE determination and ELISA-inhibition assays using a pool or individual sera from 26 patients with baker's asthma. Thermal denaturation and simulated gastrointestinal digestion of both Tri a 14 forms were checked by spectroscopic and electrophoretic methods, respectively, and biological activity by basophil activation test (BAT).

Results Natural and recombinant Tri a 14 were similarly folded, as indicated by their nearly identical CD spectra and heat denaturation profiles. A high interclass correlation coefficient [0.882] was found between specific IgE levels to both Tri a 14 proteins in individual sera from baker's asthma patients, but a slightly lower IgE-binding potency of rTri a 14 was detected by ELISA-inhibition assays. Natural and recombinant Tri a 14 elicited positive BAT in two and one out of three patients, respectively. Heat denaturation profiles and simulated gastrointestinal digestion assays indicated that Tri a 14 displayed a high heat and digestive proteolytic resistance, comparable to those of peach Pru p 3, the model food allergen of the LTP family.

Conclusions Recombinant Tri a 14 is a potential tool for baker's asthma diagnosis, based on its physicochemical and immunological similarity with its natural counterpart. Wheat Tri a 14 shows a high thermal stability and resistance to gastrointestinal digestion.
\end{abstract}

Keywords baker's asthma, lipid transfer protein, molecular diagnosis, proteolytic resistance, recombinant allergen, wheat

ledades Respiratorias (CIBERES), Madrid, Spain. 


\section{Introduction}

Progress in genetic and protein engineering has allowed for the cloning and production of recombinant forms of relevant allergenic proteins, as well as the identification of their IgE-binding molecular regions ( $\mathrm{B}$ epitopes) and their site-directed mutation to obtain hypoallergenic variants $[1,2]$. There is growing evidence that the use of panels of recombinant allergens, termed component-resolved diagnostics [3], instead of crude extracts from the allergenic sources, provides a refined molecular analysis of sensitization patterns, thus enhancing diagnosis, prediction of cross-sensitizations and strategies of immunotherapy [1-3]. However, a previous step for the use of recombinant allergens in diagnostic panels is to prove that their immunological and physicochemical properties are equivalent to those of their natural counterparts $[4,5]$.

Baker's asthma is an important occupational disease, mainly caused by an IgE-mediated allergic response to inhalation of cereal flours [6]. Specific wheat-IgE sensitization rates over $10 \%$ and prevalence around $10 \%$ for lower respiratory symptoms and asthma have been recently reported [7]. Several families of salt-soluble proteins from wheat flour [8-12], as well as prolamins (storage proteins not extractable by salt solutions) [12, 13], have been identified as potential allergens associated with bakers asthma. However, low (20-30\%) specific IgE sensitization rates have been found for most of these allergen families [10-13], and a large variability of IgEbinding patterns of wheat protein has been observed among individual sera from patients with baker's asthma [14]. Thus, it remains uncertainty as to which proteins are the major allergens in this allergic disorder [6]. Furthermore, great differences in the concentration of potential allergens in commercial wheat diagnostic extracts have been reported [15]. Overall, this scenario warrants necessity to improve the diagnostic tools (panel of purified allergens) for baker's asthma.

Several allergens linked to this occupational disease, such as peroxidase [9], thioredoxin [10], serine proteinase inhibitor [11] and $\alpha \beta$ gliadin [13], have been isolated and tested as purified natural or recombinant proteins. However, their use as molecular markers for component resolved diagnosis presents three main constrains: specific IgE sensitization rates below $50 \%$, lack of in vivo (i.e. STP) data, and a limited number of sera analysed (from 10 to 22 patients, except in the case of $\alpha \beta$ gliadin). Taking into account this situation, wheat non-specific lipid transfer protein (LTP) Tri a 14, recently identified as a major allergen associated with baker's asthma [16], seems to be a good candidate to include in a diagnostic panel. It is supported by the sensitization rate $(60 \%$ of sera with specific IgE to the allergen) and in vivo [skin prick test (SPT)]-positive responses (in 15 out of 24 subjects; $62 \%$ ) found with purified natural Tri a 14 in a group of 40 Spanish patients with baker's asthma. Moreover, Tri a 14 has been also described as a main allergen linked to wheat food (ingestion) allergy [17], and causing cross-reactivity with relevant food allergens belonging to the LTP panallergen family, as peach Pru p $3[16,18]$. Interestingly, preliminary data point to a lower resistance of Tri a 14 to heat treatment than other allergenic LTPs, such as maize Zea m 14 [17], and structural differences between Tri a 14 and LTP allergens from maize and fruits have been suggested [19].

We reported here the cloning and production of recombinant Tri a 14, the equivalent physicochemical and immunological properties of its natural and recombinant forms, and the resistance of Tri a 14 to heat and gastrointestinal digestion.

\section{Materials and methods}

\section{Patients and sera}

Twenty-six patients with baker's asthma caused by wheat flour inhalation were selected at the Servicio de Alergia, Hospital La Paz (Madrid, Spain; numbers 1-10 in Table 1) and Fundacion Jimenez Diaz (Madrid, Spain; numbers 11-26 in Table 1). All selected patients had: (1) a convincing clinical history of occupational asthma provoked by inhalation of wheat flour in the workplace; (2) an SPTpositive response to a homemade wheat extract [16]; (3) a specific IgE level to wheat $>0.35 \mathrm{kU} / \mathrm{L}$ (mean $12.9 \mathrm{kU} / \mathrm{L}$; range $0.4-74.6 \mathrm{kU} / \mathrm{L}$ ), as measured by the Phadia CAP System FEIA (Phadia, Uppsala, Sweden); and (4) a positive specific inhalation challenge test to wheat flour, using a method previously described [20]. Demographic and clinical data of selected patients are summarized in Table 1. Written informed consent was obtained from all patients, and the study was approved by the ethics committees of Hospital La Paz and Fundacion Jimenez Diaz (Madrid, Spain).

A pool of sera from all 26 selected patients was used for IgE immunodetection and ELISA-inhibition assays, and individual sera were tested in specific IgE determination assays to natural and recombinant Tri a 14 and to recombinant peach Pru $\mathrm{p} 3$.

Isolation of the cDNA encoding Tri a I4 and expression of the recombinant allergen in Pichia pastoris

Total RNA from wheat bran was obtained by the method of Chang et al. [21]. The reverse transcription was carried out with $2 \mu \mathrm{g}$ of total RNA using the oligonucleotide primer 5'-GATGGATGAGCTGAGCACCAGCTTG-3' and the High Capacity cDNA Reverse Transcription Kit (Applied Biosystems, Foster City, CA, USA). The cDNA obtained was amplified by polymerase chain reaction 
Table 1. Demographic data and specific IgE levels to wheat and purified natural (n) and recombinant (r) Tri a 14 and peach rPru p 3 of the selected patients with baker's asthma

\begin{tabular}{|c|c|c|c|c|c|}
\hline \multirow[b]{2}{*}{ Patient no. } & \multirow[b]{2}{*}{ Age (years)/sex } & \multirow{2}{*}{$\begin{array}{l}\text { Specific [gE to } \\
\text { wheat (CAP, kU/L) }\end{array}$} & \multicolumn{3}{|c|}{ Specific IgE to ${ }^{*}$ (ELISA, OD unit) } \\
\hline & & & nTri a 14 & rTri a 14 & IPru p 3 \\
\hline 1 & $34 / \mathrm{M}$ & 3.0 & 0.17 (neg.) & 0.20 (neg.) & 0.05 (neg.) \\
\hline 2 & $48 / \mathrm{M}$ & 8.2 & 0.16 (neg.) & 0.31 & 0.05 (neg.) \\
\hline 3 & $50 / \mathrm{M}$ & 4.9 & 0.25 & 0.29 & 0.05 (neg.) \\
\hline 4 & $48 / \mathrm{M}$ & 0.5 & 0.26 & 0.26 & 0.09 (neg.) \\
\hline 5 & $49 / \mathrm{M}$ & 3.1 & 0.24 & 0.26 & 0.25 \\
\hline 6 & $35 / \mathrm{M}$ & 8.5 & 0.67 & 0.70 & 0.04 (neg.) \\
\hline 7 & $41 / \mathrm{M}$ & 0.4 & 0.18 (neg.) & 0.15 (neg.) & 0.06 (neg.) \\
\hline 8 & $43 / \mathrm{M}$ & 35.8 & 0.32 & 0.34 & 0.04 (neg.) \\
\hline 9 & $44 / \mathrm{F}$ & 6.4 & 0.16 (neg.) & 0.14 (neg.) & 0.03 (neg.) \\
\hline 10 & $53 / \mathrm{F}$ & 1.2 & 0.32 & 0.23 & 0.26 \\
\hline 11 & $35 / \mathrm{M}$ & 74.2 & 0.19 (neg.) & 0.25 & 0.07 (neg.) \\
\hline 12 & $29 / \mathrm{M}$ & 18.0 & 0.58 & 0.83 & 0.32 \\
\hline 13 & $36 / \mathrm{M}$ & 1.7 & 0.32 & 0.37 & 0.04 (neg.) \\
\hline 14 & $39 / \mathrm{M}$ & 25.8 & 0.30 & 0.33 & 0.26 \\
\hline 15 & $28 / \mathrm{M}$ & 2.6 & 0.26 & 0.13 (neg.) & 0.03 (neg.) \\
\hline 16 & $27 / \mathrm{M}$ & 7.9 & 0.14 (neg.) & 0.16 (neg.) & 0.09 (neg.) \\
\hline 17 & $26 / \mathrm{M}$ & 2.4 & 0.26 & 0.25 & 0.26 \\
\hline 18 & $54 / \mathrm{M}$ & 2.5 & 0.12 (neg.) & 0.15 (neg.) & 0.04 (neg.) \\
\hline 19 & $42 / \mathrm{F}$ & 3.7 & 0.22 & 0.18 (neg.) & 0.26 \\
\hline 20 & $54 / \mathrm{M}$ & 1.7 & 0.11 (neg.) & 0.11 (neg.) & 0.25 \\
\hline 21 & $26 / \mathrm{M}$ & 74.6 & 0.34 & 0.35 & 0.28 \\
\hline 22 & $31 / \mathrm{M}$ & 0.8 & 0.25 & 0.21 (neg.) & 0.24 \\
\hline 23 & $43 / \mathrm{M}$ & 13.8 & 0.12 (neg.) & 0.12 (neg.) & 0.05 (neg.) \\
\hline 24 & $34 / \mathrm{F}$ & 2.0 & 0.24 & 0.25 & 0.16 (neg.) \\
\hline 25 & $21 / \mathrm{F}$ & 19.0 & 0.25 & 0.15 (neg.) & 0.07 (neg.) \\
\hline 26 & $35 / \mathrm{F}$ & 1.9 & 0.12 (neg.) & 0.15 (neg.) & 0.13 (neg.) \\
\hline
\end{tabular}

"Values are means $(n=3)$. OD $\leqslant 0.21$ units (mean [OD units, $n=12$ ] \pm 3 SD to BSA used as negative control; $0.081+3 \times 0.043=0.21$ ).

neg, negative value.

(PCR) using the oligonucleotide $5^{\prime}$ primer: $5^{\prime}$-TACGGT GCACTGCACTGTTAGCTACAGACC- $3^{\prime}$ and $3^{\prime}$ primer: $5^{\prime}-$ GATGGATGAGCTGAGCACCAGCTTG- $3^{\prime}$, designed from LTP cDNAs previously reported [22]. The amplification was carried out with Taq-Gold DNA polymerase (PerkinElmer, Branchburg, NY, USA) as recommended by the manufacturer. The PCR program was 5 min denaturation at $94^{\circ} \mathrm{C}$, followed by 30 cycles each of $30 \mathrm{~s}$ denaturation at $94^{\circ} \mathrm{C}, 1 \mathrm{~min}$ annealing at $68^{\circ} \mathrm{C}$, and $2 \mathrm{~min}$ at $72^{\circ} \mathrm{C}$, and final extension for $7 \mathrm{~min}$ at $72^{\circ} \mathrm{C}$. The PCR products were cloned into pGEM T-Easy vector (Promega, Madison, WI, USA), and these constructs were used to transform Escherichia coli XL1-Blue cells. The DNAs from several clones were sequenced using the ABI PRISM Dye Terminator kit and an ABI 3100 sequencer (Perkin-Elmer Biosystem, Warrington, UK).

The encoding region of the Tri a 14 cDNA was amplified by PCR with Taq-Gold DNA polymerase (Perkin-Elmer), a sense primer $5^{\prime}$-CCCTCGAGAAAAGAGAGGCTGAAATC GACTGCGGCCACG-3' (XhoI restriction site underlined) and an antisense $5^{\prime}$-CGGAATTCTTACACCCTGCTGCAG TCGATG-3' (EcoRI restriction site underlined). The sense primer allows fusion of the Tri a 14 encoding region inframe with the sequence coding for the pre-prosequence of the Saccharomyces cerevisiae $\alpha$ factor present in plasmid pPIC9. This PCR product was purified, digested with $X h o l / E c o R I$ restriction enzymes and inserted into the same sites of the pPIC9 vector to transform XL1-Blue $E$. coli, and the DNA from several clones was sequenced to confirm the in-frame arrangement described above.

Ten micrograms of pPIC9/Tri a 14 were digested with $B g$ III restriction enzyme and the larger purified fragment was used to transform GS115 P. pastoris cells by electroporation following the Invitrogen manual recommendations (Invitrogen Corporation, De Schelp, the Netherlands). Transformed cells were grown on minimal glucose plates at $30^{\circ} \mathrm{C}$ during 3-4 days, and screening for positive clones was performed by patching the colonies in replica-plating on minimal glucose vs. minimal methanol plates. The selected transformants were cultured in buffered glycerol minimum medium during $48 \mathrm{~h}$ at $30^{\circ} \mathrm{C}$. Cells were then collected by centrifugation, and expression of the recombinant protein was induced by resuspension in buffered methanol minimum medium. 


\section{Purification of recombinant and natural Tri a 14}

The supernatants from transformed $P$. pastoris cultures obtained after induction were dialysed against $0.1 \mathrm{M}$ ammonium acetate, $\mathrm{pH} 6.8$ and freeze-dried. This preparation was fractionated by gel filtration on a Superdex HR 75 16/60 column (Pharmacia Biotech, Uppsala, Sweden), eluting with $0.1 \mathrm{M}$ ammonium acetate, $\mathrm{pH} 6.8(1 \mathrm{~mL} /$ min). Fractions enriched in rTri a 14 were pooled, freezedried and then separated by RP-HPLC on a Nucleosil 300C4 column $(8 \times 250 \mathrm{~mm}$; particle size $5 \mu \mathrm{m}$; Scharlau Science, Barcelona, Spain). Elution was performed with a gradient of acetonitrile in $0.1 \%$ trifluoroacetic acid ( $10 \%$ during $5 \mathrm{~min}$, and $10-100 \%$ in $150 \mathrm{~min} ; 1 \mathrm{~mL} / \mathrm{min}$ ). Chromatographic peaks containing rTri a 14 were identified by SDS-PAGE separation followed by immunodetection with anti-Pru $\mathrm{p} 3$ rabbit polyclonal antibodies or a serum pool from baker's asthma patients.

Natural Tri a 14 was isolated as previously described [16].

The isolated nTri a 14 and rTri a 14 were quantified by a commercial bicinchoninic acid test (Pierce, Cheshire, UK). Purity of both protein preparation was tested by SDSPAGE, N-terminal amino acid sequencing with an Applied Biosystems Procise 494 gas-phase sequencer, and matrixassisted laser desorption/ionization (MALDI) mass spectrophotometric analysis with a Biflex III Spectrometer (Bruker-Franzen Analytik, Bremen, Germany), using standard methods.

\section{Circular dichroism and thermal denaturation spectroscopy}

Circular dichroism (CD) measurements were performed on a Jasco Model J-720 Spectropolarimeter (Japan Spectroscopic Co., Tokyo, Japan) using a quartz cell with a $1 \mathrm{~mm}$ light path length, thermostatically controlled with a Jasco Model 423S Peltier-type temperature controller, and a thermostated cell holder, interfaced with a thermostatic bath. The far-UV spectra were recorded at $20^{\circ} \mathrm{C}$ from 190 to $260 \mathrm{~nm}$, as an average of five scans after being corrected by sutration of a buffer blank with the following parameters: scan speed, $50 \mathrm{~nm} / \mathrm{min}$; bandwidth, $1 \mathrm{~nm}$; response time, $4 \mathrm{~s}$; and step resolution, $0.2 \mathrm{~nm}$. Thermal denaturation data were recorded in the temperature range $5-90^{\circ} \mathrm{C}$ at $220 \mathrm{~nm}$ by the following step scan procedure: heating rate, $1^{\circ} \mathrm{C} / \mathrm{min}$; response, $4 \mathrm{~s}$; recorder interval, $0.2^{\circ} \mathrm{C}$. The protein concentration used were 17.3 $\mu_{\mathrm{M}}$ for natural Pru p 3, 17.7 $\mu_{\mathrm{M}}$ for natural Tri a 14 and $13.0 \mu \mathrm{m}$ for recombinant Tri a 14 , as quantified by using amino acid analysis after acid hydrolysis. All proteins were in $65 \mathrm{~mm}$ potassium phosphate buffer, $\mathrm{pH}$ 7.2. Optical activity was expressed as mean residue ellipticity $[\theta]$, in degrees $\mathrm{cm}^{2} / \mathrm{dmol}$, based on the calculated mean residue weight of each protein. Analysis of protein secondary structure from $C D$ spectra was performed using the CDSSTR program included at the DICHROWEB site (http://public-1.cryst.bbk.ac.uk/cdweb/ html) [23].

\section{Immunodetection assays}

Samples (15 $\mu$ g of protein from supernatants of induced and not induced $P$. pastoris transformed cultures, and $3 \mu \mathrm{g}$ of purified nTri a 14 and rTri a 14) were fractionated by SDS-PAGE on Bio-Rad Miniprotean III System gels (15\% polyacrylamide) (Bio-Rad Laboratories, Hercules, CA, USA), according to the method of Laemmli [24]. The fractionated samples were then electrotransferred onto polyvinilidene difluoride membranes. After blocking, membranes were incubated overnight with a pool of sera from the 26 selected patients with baker's asthma (1:5 dilution), washed and incubated with goat anti-human IgE-peroxidase conjugate (Biosource, Camarillo, CA, USA) ( $1: 3000$ dilution; $1 \mathrm{~h}$ ). Detection of IgE-binding components was achieved by means of chemiluminiscence, according to the manufacturer's instructions (Amersham Biosciences, Little Chalfont, UK). A pool of sera (1:5 dilution) from bakers non-allergic to wheat (negative IgE and SPT responses and non symptoms) was tested as a negative control [16]. No band was recognized by this control serum pool in any of the samples tested.

Alternatively, replica gels were immunodetected with rabbit polyclonal antibodies to the peach allergen Pru p 3 ( $1: 500$ dilution), and then treated with goat anti-rabbit IgG peroxidase-conjugated antibody (1:5000 dilution; Sigma, SteinHeim, Germany), and revealed as described above.

\section{Specific IgE determination and ELISA-inhibition assays}

ELISAs to determine specific IgE to nTri a 14 and rTri a 14 ( $5 \mu \mathrm{g} / \mathrm{mL}$ as solid phase) of the 26 individual sera (1:5 dilution) from patients with baker's asthma, were performed as previously described [5]. BSA $(5 \mu \mathrm{g} / \mathrm{mL})$ and a serum pool from subjects $(n=10)$ allergic to dust mites, but not plant foods or pollens, were used as negative controls. Specific IgE levels were considered to be positive when $>0.21$ OD units (see Table 1). All tests were performed in triplicate.

ELISA-inhibition assays were carried out by the same method, except that the pool of sera was comprised of those individual sera $(n=18)$ with specific IgE to nTri a 14 and/or rTri a 14 (see Table 1), and was previously incubated with $10,5,2$ and $1 \mu \mathrm{g} / \mathrm{mL}$ of the natural or recombinant Tri a 14 forms as inhibitor $\left(3 \mathrm{~h}\right.$ at $25^{\circ} \mathrm{C}$. All tests were performed in triplicate.

\section{Basophil activation tests}

rTri a 14, nTri a 14 and a wheat salt-soluble protein preparation were tested at $0.1-1 \mu \mathrm{g} / \mathrm{mL}$ for activation of 
basophils from three whole blood samples of baker asthmatic donors and one atopic non-asthmatic donor based on analysis of up-regulation of surface expression of CD63 on IgE-positive basophils by flow cytometry [25]. The tests were considered positive when the stimulation index ( $\%$ of activated basophils with allergen $/ \%$ of activated basophils at baseline conditions) was $\geq 5$.

\section{Simulated gastric and duodenal digestion}

Digestion of purified nTri a 14, rTri a 14 and nPru p 3 by simulated gastric fluid (SGF) was performed essentially as described by Diaz-Perales et al. [26]. The purified allergens $(1 \mu \mathrm{g} / \mu \mathrm{L})$ were dissolved in SGF solution $(100 \mathrm{~mm} \mathrm{HCl}, \mathrm{pH}$ $1.2,30 \mathrm{~mm} \mathrm{NaCl}$ ), containing $0.32 \%(w / v)$ of pepsin A (Calbiochem, Darmstadt, Germany). Digestion was carried out at $37^{\circ} \mathrm{C}$ during $120 \mathrm{~min}$. Control samples without pepsin were included.

The in vitro duodenal digestion was achieved following the method described by Moreno et al. [27]. The freezedried samples resulting after $120 \mathrm{~min}$ of gastric digestion were dissolved in simulated duodenal solution $(0.125 \mathrm{M}$ bile salt mix of sodium taurocholate and glycodeoxycholic acid (Calbiochem), $1 \mathrm{M} \mathrm{CaCl} 2,0.25 \mathrm{M}$ Tris, pH 6.5; porcine pancreatic lipase $(0.1 \%$; Calbiochem); porcine colipase $(0.055 \% \mathrm{w} / \mathrm{v}$; Calbiochem); trypsin $(0.1 \% \mathrm{w} / \mathrm{v}$; Sigma); and $\alpha$-chymotrypsin $(0.4 \% \mathrm{w} / \mathrm{v}$; Sigma). The digestion was carried out at $37^{\circ} \mathrm{C}$ and aliquots were taken at $0,2,30$ and $120 \mathrm{~min}$. The reaction was stopped by heating at $80^{\circ} \mathrm{C}$ during $5 \mathrm{~min}$, and samples were then freeze-dried. Control samples without trysin and $\alpha$-chymotrypsin were included.

Samples taken at different stages of digestion $(5 \mu \mathrm{L})$ were analysed by SDS-PAGE on 12\% polyacrylamide NuPAGE ${ }^{\circledR 1}$ Novex Bis-Tris precast gels (Invitrogen, Carlsbad, CA, USA), and Coomassie R125 stained.

\section{Statistical analysis}

The Student $t$-test was used to compare the specific IgE levels to nTri a 14 and rTri a 14 in 26 individual sera from patients with baker's asthma. Correlation between specific IgE levels to the different allergens tested was analysed by one-way intraclass correlation coefficient (ICC). The comparison and the correlation were performed at a signifi- cant level of $P<0.05$. All test were performed using SPSS statistical software version 12 for Windows (SPSS Inc., Chicago, IL, USA).

\section{Results}

\section{Production and characterization of recombinant Tri a 14}

Natural Tri a 14 was isolated from a wheat bran extract by means of a two-step chromatographic procedure previously described [16]. Purity of the protein was ascertained by a single SDS-PAGE band of the expected apparent molecular weight (Fig. 1a), a single N-terminal amino acid sequence (IDXGHVDSLVR), which completely matched that reported by Palacin et al. [16], and a major sharp peak at $9667 \mathrm{Da}$ in MALDI analysis.

Based on the determined N-terminal amino acid sequence, together with the nucleotide sequences of cDNA clones encoding wheat LTPs [22], specific oligonucleotide primers were designed to identify the full cDNA corresponding to Tri a 14. Characterization of the selected clone rendered a nucleotide sequence (Fig. 2) identical to that of the cDNA clone Ta Ltp9.1a [22] (accession no. AJ852536), which encodes a LTP isoform highly expressed in hexaploid (bread) wheat seed. The correspondence of purified natural Tri a 14 and the amino acid sequence deduced from the selected cDNA clone (Fig. 2) was supported by their identical $\mathrm{N}$-terminal sequence and compatible molecular mass $(9607 \mathrm{Da}$ calculated from the amino acid sequence shown in Fig. 2).

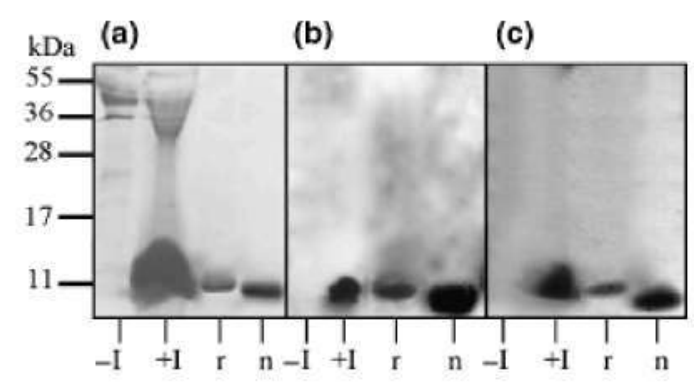

Fig. 1. Crude extracts from supernatants of induced $(+\mathrm{I})$ and not induced (-I) Pichia pastoris yeast cultures transformed with pPIC9/Tri a 14 vector, and purified natural (n) and recombinant (r) Tri a 14, were separated by SDS-PAGE and stained with Coomassie Blue (a), or immunodetected with anti-Pru p 3 rabbit polyclonal antibodies (b) or with a serum pool from baker's asthma patients (c).



Fig. 2. Nucleotide sequence and deduced amino acid sequence of the selected cDNA clone encoding Tri a 14. 
(a)

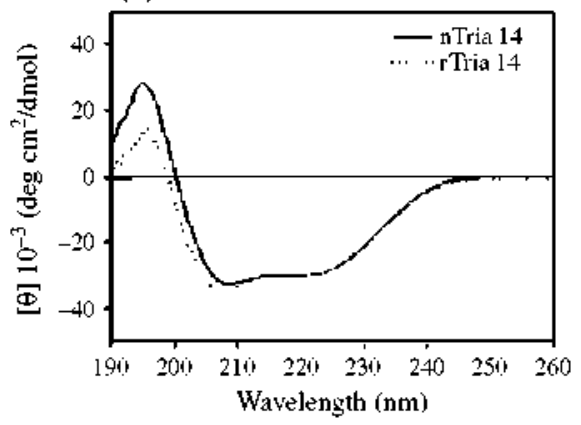

(b)

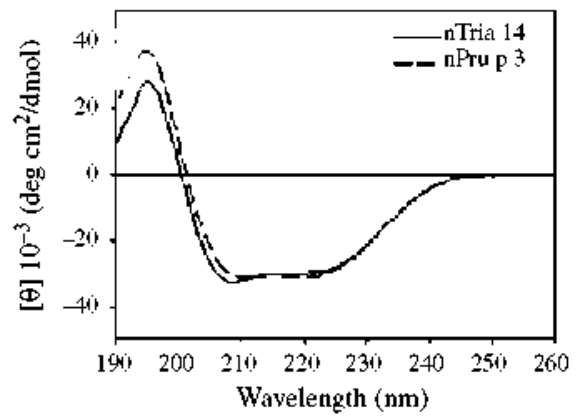

Fig. 3. Comparison of circular dichroism spectra of natural (n) and recombinant (r) Tri a 14 (a), and natural Tri a 14 and peach Pru p 3 (b). Samples of purified proteins (17.7-13 $\mathrm{ms}_{\mathrm{s}}$ in $65 \mathrm{~mm}$ potassium phosphate buffer, $\mathrm{pH} 7.2$, were analysed at $20^{\circ} \mathrm{C}$.

To produce the recombinant allergen, $P$. pastoris GS115 cells were transformed with pPIC9 expression plasmid carrying the Tri a $14 \mathrm{cDNA}$ (pPIC9/Tri a 14) and induced with methanol (Fig. 1). The expressed rTri a 14 was then purified from supernatants of the transformed yeast cultures by gel-filtration chromatography followed by reverse-phase HPLC. Proper expression and purification were confirmed by the presence of a single band in SDSPAGE, its recognition by rabbit polyclonal antibodies to Pru p 3, the major LTP allergen from peach fruit, and the binding of rTri a 14 to IgE from sera of patients with baker's asthma (Fig. 1). Furthermore, the isolated recombinant allergen showed a single $\mathrm{N}$-terminal amino acid sequence (EAEIDXGHVD) corresponding to that of the natural form, but with three (EAE) extra residues at the $\mathrm{N}$-terminus by an alternative processing of the signal peptide of $P$. pastoris, and a molecular mass of $9913 \mathrm{Da}$ in MALDI analysis, in agreement with that calculated from its putative full amino acid sequence $(9936 \mathrm{Da})$.

\section{Natural and recombinant Tri a 14 present equinalent secondary structure patterns and heat resistance}

Comparisons of the far-UV spectra at $20^{\circ} \mathrm{C}$ of natural and recombinant Tri a 14, as well as natural Tri a 14 and peach Pru p 3, selected as model of LTP allergens from plant foods [18], are presented in Fig. 3. No difference between nTri a 14 and rTri a 14 was detected, suggesting that the native structure of the recombinant form is essentially the same as that of the natural allergen. Similar CD spectra were also found for $n T r i$ a 14 and nPru p 3 . The strong structural similarity among the three allergens was further confirmed by secondary structure calculations (Table 2). Optimal good fittings parameters NRMSD (normalized root mean square deviation), issued from the comparison between experimental and back-calculated data (SP43 reference set), were obtained with the CDSSTR program $[23,28]$, reflecting the accurate analysis of secondary structure (NRMSD $\leqslant 0.002$ in all cases). The secondary
Table 2. Secondary structure composition of natural Trì a 14 and Pru p 3 , and recombinant Tri a 14 allergens, as calculated from their far- $U V$ CD data

\begin{tabular}{|c|c|c|c|c|c|}
\hline \multirow[b]{2}{*}{ Allergen } & \multicolumn{4}{|c|}{ Secondary structure $(\%)$} & \multirow[b]{2}{*}{ NRMSD } \\
\hline & $\alpha$-helix & $\beta$-sheet & $\beta$-turn & Unordered & \\
\hline nTri a 14 & 70 & 10 & 9 & 11 & 0.001 \\
\hline rTrì a 14 & 66 & 13 & 6 & 15 & 0.002 \\
\hline nPru p 3 & 71 & 12 & 7 & 10 & 0.001 \\
\hline
\end{tabular}

CD, circular dichroism; NRMSD, normalized root mean square deviation.

structure found for both Tri a 14 and Pru $\mathrm{p} 3$ is dominated by $\alpha$-helices $(\sim 70 \%)$, in agreement with the known $\mathrm{X}$-ray structures of these allergens $[29,30]$.

The thermal unfolding of nTri a 14, rTri a 14 and nPru $p$ 3 was monitored by $\mathrm{CD}$, measuring changes in molar ellipticity at $220 \mathrm{~nm}$ upon heating (up to $90^{\circ} \mathrm{C}$; see Fig. 4). Similar scans were obtained for the three allergens with non-unfolding transition in the range $5-90^{\circ} \mathrm{C}$. Analysis of the temperature progress unfolding curves reveals that the native conformations of all three proteins were thermally stable up to $70^{\circ} \mathrm{C}$, with minimum partial loss of their $\alpha$ helical structure. The secondary structure was only partially perturbed upon heating to $90^{\circ} \mathrm{C}$, but even retaining most of their native state $\alpha$-helical content $(77 \%, 82 \%$ and $51 \%$ for nTri a 14 , rTri a 14 and nPru p 3, respectively). Overall, these results indicated that these allergens display a high thermal stability.

\section{Natural and recombinant Tri a 14 show similar IgE-binding capacity}

Besides their equivalent structural and heat denaturation behaviour, it should be also established that nTri a 14 and rTri a 14 exhibited similar IgE-binding capacity. A complementary dual experimental approach led to cover this requirement. Specific IgE determination to both allergen forms was performed in 26 individual sera from patients 
(a)

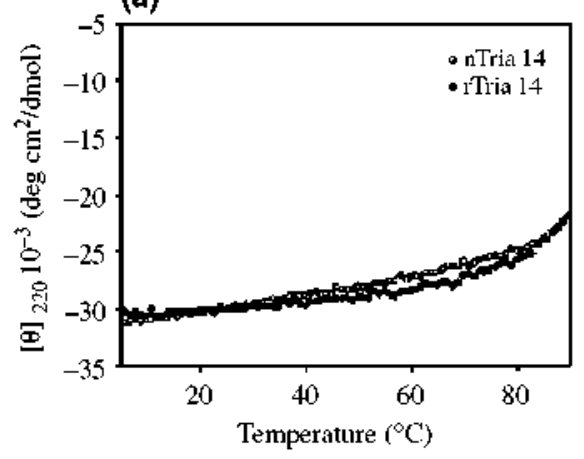

(b)

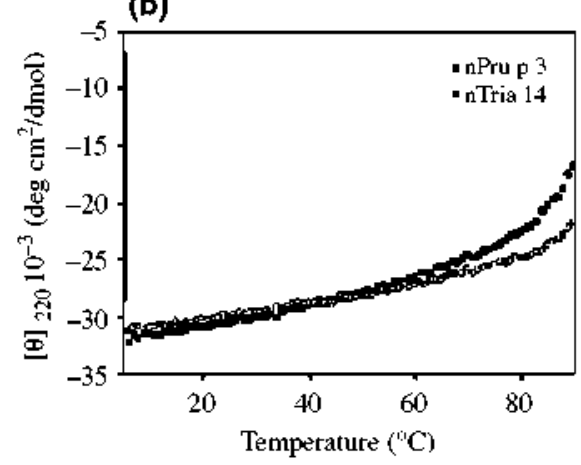

Fig. 4. Comparison of thermal denaturation profiles of natural (n) and recombinant (r) Trì a 14 (a), and natural Trì a 14 and peach Pru p 3 (b), as monitored by changes in ellipticity at $220 \mathrm{~nm}$.
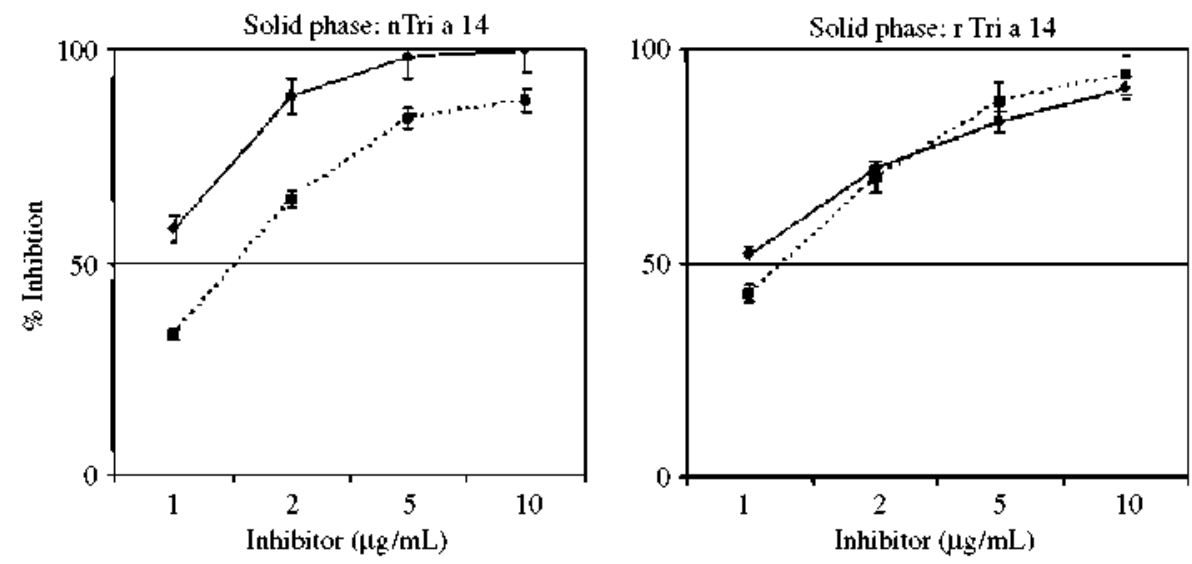

$\longrightarrow$ n Tri a $14 \quad$... 1 Tri a 14

Fig. 5. ELISA-inhibition with natural (n) and recombinant (r) Trì a 14 in the solid-phase and as inhibitors. Means ( $n=3$ ) and SDs (bars) are represented.

with baker's asthma (Table 1). Positive assays were observed in $61 \%(16 / 26)$ and $54 \%(14 / 26)$ of sera for nTri a 14 [mean (OD units) \pm SD: $0.25 \pm 0.13$ ] and rTri a 14 [mean (OD units) \pm SD: $0.26 \pm 0.17$ ], respectively. A strong correlation was found between the levels of specific IgE to both recombinant and natural Tri a 14 (ICC $=0.882 ; P<0.001$ ). By contrast, only $34 \%$ (9/26) of sera showed specific IgE to peach rPru p 3 (Table 1), and no correlation was detected between the IgE levels to this allergen and those to nTri a 14 or rTri a 14 (ICC $=-0.014$ and -0.003 , respectively; $P>=0.05$ in both cases). In addition, ELISA-inhibition assays (Fig. 5) reflected a similar inhibitory behaviour of both allergen forms, but uncovering a slightly lower potency of rTri a 14 (allergen concentration to reach 50\% inhibition: (a) nTri a 14 in solid phase: 0.86 and $1.50 \mu \mathrm{g} /$ $\mathrm{mL}$ for nTri a 14 and rTri a 14, respectively; (b) rTri a 14 in solid phase: 0.96 and $1.20 \mu \mathrm{g} / \mathrm{mL}$ for nTri a 14 and rTri a 14, respectively).

Basophil activation tests (BATs) supported the biological activity of the natural and recombinant allergen (Table 3). Two and one out of three patients with baker's asthma showed positive responses to nTri a 14 and rTri a 14 , respectively.
Table 3. Maximum basophil activation responses induced by a wheat salt soluble protein extract (WE), nTrì a 14 and rTrí a 14 in blood from patients with baker's asthma

\begin{tabular}{lllll}
\hline & & \multicolumn{3}{l}{ \% activated basophils (SI) } \\
\cline { 3 - 5 } & Time (years) & WE & nTri a 14 & rTri a 14 \\
Subject no. & without contact $^{\dagger}$ & $(1 \mu \mathrm{g} / \mathrm{mL})$ & $(1 \mu \mathrm{g} / \mathrm{mL})$ & $(0.1 \mu \mathrm{g} / \mathrm{mL})$ \\
\hline I & 3 & $49.2(259.2)$ & $46.6(245.4)$ & $34.7(183.0)$ \\
II & 5 & $28.4(25.1)$ & $31.3(27.8)$ & $0.2(0.2)$ \\
III & 5 & $28.5(150.0)$ & $0.5(2.8)$ & $0.8(4.5)$ \\
C & - & $0.9(1.6)$ & $0.4(0.8)$ & $1.0(1.7)$
\end{tabular}

"Subjects It II and III are patients with baker's asthma caused by wheat flour inhalation and subject $C$ is an atopic patients without baker's asthma.

"Years without contact with inhaled wheat flour.

${ }^{\ddagger}$ Maximum activation values. $S I \geqslant 5$ was considered a positive response. SI, stimulation index.

Both natural and recombinant Tri a 14 are resistant to simulated gastric and duodenal digestion

The behaviour of recombinant Tri a 14 to proteolytic enzymes, compared with that of nTri a 14 and nPru p 3, 


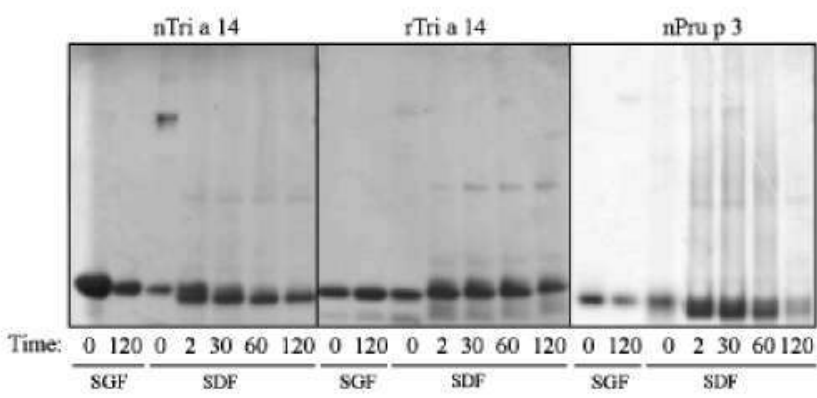

Fig. 6. Simulated gastric followed by duodenal digestion of natural (n) and recombinant $(\mathrm{r})$ Tri a 14 , and natural Pru p 3 . Samples $(5 \mu \mathrm{g})$ were fractionated by SDS-PAGE after $120 \mathrm{~min}$ of simulated gastric digestion (SGF) or 2, 30, 60, or $120 \mathrm{~min}$ of a following simulated duodenal digestion (SDF). Time 0 represents control samples in SGF without pepsin, or SDF without trypsin and $\alpha$-chymotrypsin.

was checked in SGF followed by duodenal digestion fluid (SDF) (Fig. 6). All three purified allergens were mostly resistant to both SGF (120 $\mathrm{min})$ and SDF (120 min after SGF treatment), thus indicating that both forms of Tri a 14 (natural and recombinant) probably remain immunologically active during gastrointestinal digestion.

\section{Discussion}

In spite of the relevance of baker's asthma as occupational disease, no panel of molecular markers to diagnose this allergic disorder have been developed. The low sensitization rates (as estimated by specific IgE) to most identified allergens associated with this disease, together with the limited number of patients studied in each case [9-13], and the reported variability in sensitization patterns [14], have probably retarded the development of such molecular panel. In contrast, specific IgE to $\omega-5$ gliadin has been proposed as a useful tool for diagnosis of wheatdependant exercise-induced anaphylaxis and wheat food allergy [31, 32].

Tri a 14, an abundant LTP in wheat seeds, has been recently identified by in vitro and in vivo methods as a major allergen associated with baker's asthma in Spanish patients [16]. Additionally, it seems to be also relevant in wheat IgE-mediated food allergy in Italian subjects [17]. This background suggests that Tri a 14 could be a useful tool for diagnosis of wheat allergy, at least in the Mediterranean population. Accordingly, as a preliminary step, the cDNA coding for Tri a 14 was isolated and its recombinant form expressed in the yeast $P$. pastoris to produce high and homogeneous amounts of such a tool, and to allow its future manipulation by protein engineering. The produced rTri a 14 was isolated from supernatants of transformed yeast cultures, and its purity and correspondence with the natural allergen confirmed by SDSPAGE and MALDI analysis, and N-terminal amino acid sequencing, that detected the presence of three additional residues (EAE) with respect to the natural form. These extra residues do not seem to impair the equivalence of both allergen versions, but could weakly decrease the IgE-binding capacity of the recombinant form, as discussed below.

The use of the recombinant form of an allergen as a potential diagnostics tool, previously requires to ascertain the similarity of its biophysical and immunological properties to those of its natural counterpart. The almost identical CD spectra and heat denaturation profiles of nTri a 14 and rTri a 14 support their physicochemical equivalence, as well as the proper expression of the recombinant protein. On the other hand, two lines of evidence point to the similar IgE-binding capacity of both Tri a 14 forms. Specific IgE determination uncovered equivalent qualitative and quantitative responses ( $61 \%$ vs. $54 \%$ positive sera and mean values of 0.25 vs. $0.26 \mathrm{OD}$ units for nTri a 14 and rTri a 14, respectively), which render a statistically significant correlation (ICC $=0.882 ; P<0.001$ ) between the levels of specific IgE to each allergen version in individual sera from patients with baker's asthma. Results of ELISA-inhibition assays reflected also a comparable behaviour against specific IgE of the natural and recombinant Tri a 14, but a slightly lower potency $(\sim 20 \%)$ of the latter form. Besides, more than $50 \%$ of the 26 individual sera analysed contain specific IgE to Tri a 14 , confirming previous data [16] on its prevalence in Spanish adult subjects with baker's asthma. Additionally, the results of BATs indicate that both allergen forms are biologically active, although also suggesting a lower activity of rTri a 14.

The experimental data presented here focus a second issue related to Tri a 14: the putative lower structural stability of this allergen than other members of the LTP panallergen family, such as maize Zea m 14, as well as its potential divergent three-dimensional (3D) structure [17, 18]. nPru p 3, the major fruit peach allergen and prototypic member of the plant food LTP family involved in allergic reactions [18], was tested here as a reference model. Comparison of the $\mathrm{CD}$ and thermal denaturation spectra of natural Tri a 14 and Pru p 3 indicates that both LTP allergens showed a very similar secondary structure and probably 3D folding (confirmed by the experimental data previously reported [29, 30]), as well as thermal resistance. In fact, important changes in secondary structure were only detected over $90^{\circ} \mathrm{C}$, thus highlighting the high thermal stability of both wheat and peach allergens. Moreover, a strong resistance to simulated gastrointestinal digestion has been detected for Tri a 14, similar to that of Pru p 3, and in line with those found for other allergenic LTPs $[18,33,34]$. Resistance of wheat LTP to simulated gastric digestion using a different experimental protocol, as well as its Caco-2 cell line translocation capability, have been previously demonstrated [35]. Therefore, it does seem that the wheat LTP allergen displays neither lower 
structural stability nor divergent 3D structure as compared with the Pru p 3 model. However, processing of wheat seeds or flour to produce derived foodstuffs could modify the Tri a 14 allergen and hardly affect both its 3D structure and IgE-binding properties [19]. In this line, different types of biochemical modifications have been described in barley LTP during the brewing process [36].

Finally, it should be mentioned that, in spite of the extensive evidence here presented supporting the physicochemical and immunological similarity of nTri a 14 and rTri a 14, to test sera from large groups of baker's asthma patients from different geographical areas is necessary to further confirm the usefulness of rTri a 14 as a tool in baker's asthma diagnosis.

\section{Acknowledgements}

We thank Dr P. Gamboa (Hospital de Basurto, Bilbao, Spain) for assistance on the statistical analysis. Financial support from Ministerio de Educacion y Ciencia-DGI (grant BI02006-07473) is also acknowledged.

\section{References}

J Bhalla PL, Singh MB. Biotechnology-based allergy diagnosis and vaccination. Trends Biotechnol 2008; 26:153-61.

2 Steckelbroeck S, Ballmer-Weber BK, Vieths S. Potential, pitfalls, and prospects of food allergy diagnostics with recombinant allergens or synthetic sequential epitopes. J Allergy Clin Immunol 2008; 121:1323-30.

3 Valenta R, Lidholm J, Niederberger V, Hayek B, Kraft D, Gronlund $\mathrm{H}$. The recombinant allergen-based concept of componentresolved diagnostics and immunotherapy (CRD and CRIT). Clin Exp Allergy 1999; 29:896-904.

4 Lorenz AR, Scheurer S, Haustein D, Vieths S. Recombinant food allergens. J Chromatogr B Biomed Sci Appi 2001; 756:255-79.

5 Diaz-Perales A. Sanz ML, Garcia-Casado G et al. Recombinant Pru p 3 and natural Pru p 3, a major peach allergen, show equivalent immunologic reactivity: a new tool for the diagnosis of fruit allergy. J Allergy Clin Immunol 2003; 111:628-33.

6 Brant A. Baker's asthma. Curr Opin Allergy Clin Immunol 2007; 7:152-5.

7 Jacobs JH, Meijster T, Meijer E, Suarthana E, Heederik D. Wheat allergen exposure and the prevalence of work-related sensitization and allergy in bakery workers. Allergy 2008; 12:1597-604.

8 Armentia A, Sanchez-Monge R, Gomez L, Baber D, Salcedo G. In vivo allergenic activities of eleven purified members of a major allergen family from wheat and barley flour. Clin Exp Allergy $1993 ; 23: 410-5$.

9 Sanchez-Monge R, Garcia-Casado G, Lopez-0tin C, Armentia A, Salcedo $G$. Wheat flour peroxidase is a prominent allergen associated with baker's asthma. Clin Exp Allergy 1997; 27:1130-7.

10 Wejchel M, Glaser AG, Ballmer-Weber BK, Schmid-Grendelmeier P. Crameri R. Wheat and maize thioredoxins: a novel crossreactive cereal allergen family related to baker's asthma. $J$ Allergy Clin Immunol 2006; 117:676-81.
11 Constantin C, Quirce S, Grote M et al. Molecular and immunological characterization of a wheat serine proteinase inhibitor as a novel allergen in baker's asthma. J Immunol 2008; 180:745 ]-60.

12 Tatham AS, Shewry PR. Allergens in wheat and related cereals. Clin Exp Allergy 2008; 38:1712-26.

13 Bittner C, Grassau B, Frenzel K, Baur X. Identification of wheat gliadins as an allergen family related to baker's asthma. J Allergy Clin Immunol 2008; 121:744-9.

14 Sander I, Flagge A, Merget R, Halder TM, Meyer HE, Baur X. Identification of wheat flour allergens by means of 2-dimensional immunoblotting. J Allergy Clin Immunol 2001; 107:907-13.

15 Sander I, Merget R, Degens PO, Goldscheid N, Bruning T, RaulfHeimsoth M. Comparison of wheat and rye flour skin prick test solutions for diagnosis of baker's asthma. Allergy 2004; 59 ;95-8.

16 Palacin A, Quirce S, Armentia A et al. Wheat lipid transfer protein is a major allergen associated with baker's asthma. $J$ Allergy Clin Immunol 2007; 120:1132-8.

17 Pastorello EA, Farioli L, Conti A et al. Wheat IgE-mediated food allergy in European patients: alpha-amylase inhibitors, lipid transfer proteins and low-molecular-weight glutenins. Allergenic molecules recognized by double-blind, placebo-controlled food challenge. Int Arch Allergy Immunol 2007; 144:10-22.

18 Salcedo G, Sanchez-Monge R, Baber D, Diaz-Perales A. Plant non-specific lipid transfer proteins: an interface between plant defence and human allergy. Biochim Biophys Acta 2007; 1771:781-91.

19 Mills EN, Mackie AR. The impact of processing on allergenicity of food. Curr Opin Allergy Clin Immunol 2008; 8:249-53.

20 Quirce S, Fernandez-Nieto M, Escudero C, Cuesta J, de Las Heras $\mathrm{M}$, Sastre J. Bronchial responsiveness to bakery-derived allergens is strongly dependent on specific skin sensitivity. Allergy 2006; 61:1202-8.

21 Chang S, Puryear J, Cairney J. A simple and efficient method for isolating RNA from pine trees. Plant Mol Biol Rep 1993; 11: 133-6.

22 Boutrot F, Guirao A, Alary R, Joudrier P, Gautier MF. Wheat nonspecific lipid transfer protein genes display a complex pattern of expression in developing seeds. Biochim Biophys Acta 2005; $1730 \div 114-25$.

23 Whitmore L, Wallace BA. DICHROWEB, an online server for protein secondary structure analyses from circular dichroism spectroscopic data. Nucleic Acids Res 2004; 32:W668-73.

24 Laemmli UK. Cleavage of structural proteins during the assembly of the head of bacteriophage T4. Nature $1970 ; 227: 680-5$.

25 Drew AC, Eusebius NP, Kenins L et al. Hypoallergenic variants of the major latex allergen $\mathrm{Hev} b 6.01$ retaining human T lymphocyte reactivity. $J$ Immunol $2004 ; 173: 5872-9$.

26 Diaz-Perales A, Blanco C, Sanchez-Monge R, Varela J, Carrillo T, Salcedo $\mathrm{G}$. Analysis of avocado allergen (Pers a 1) lgE-binding peptides generated by simulated gastric fluid digestion. J Allergy Clin Immunol 2003; 112:1002-7.

27 Moreno FJ, Mellon FA, Wickham MS, Bottrill AR, Mills EN. Stability of the major allergen Brazil nut $2 S$ albumin (Ber e 1) to physiologically relevant in vitro gastrointestinal digestion. FEBS J 2005; 272:341-52.

28 Sreerama N, Woody RW. Estimation of protein secondary structure from circular dichroism spectra: comparison of CONTIN, SELCON, and CDSSTR methods with an expanded reference set. Anal Biochem 2000; 287:252-60. 
29 Gincel E, Simorre JP, Caille A, Marion D, Ptak M, Vovelle F. Three-dimensional structure in solution of a wheat lipid-transfer protein from multidimensional $1 \mathrm{H}-\mathrm{NMR}$ data. A new folding for lipid carriers. Eur J Biochem 1994; 226:4] 3-22.

30 Pasquato N, Berni R, Folli C et al. Crystal structure of peach Pru p 3 , the prototypic member of the family of plant non-specific lipid transfer protein pan-allergens. J Mol Biol 2006; 356:684-94.

31 Matsuo H, Kohno K, Niihara H, Morita E. Specific IgE determination to epitope peptides of $(t)-5$ gliadin and high molecular weight glutenin subunit is a useful tool for diagnosis of wheat-dependent exercise-induced anaphylaxis. $J$ Immunol $2005 ; 175: 8116-22$.

32 Ito K, Futamura M, Borres MP et al. IgE antibodies to $\omega-5$ gliadin associate with immediate symptoms on oral wheat challenge in Japanese children. Allergy 2008; 63:1536-42.
33 Asero R, Mistrello G, Roncarolo D et al. Lipid transfer protein: a pan-allergen in plant-derived foods that is highly resistant to pepsin digestion. Int Arch Allergy Immunol 2000; 122: 20-32.

34 Vassilopoulou E, Rigby N, Moreno FJ et al. Effect of in vitro gastric and duodenal digestion on the allergenicity of grape lipid transfer protein. J Allergy Clin Immunol 2006; 118:473-80.

35 Bodinier M, Legoux MA, Pineau F et al. Intestinal translocation capabilities of wheat allergens using the Caco- 2 cell line. J Agric Food Chem 2007; 55:4576-83.

36 Perrocheau L, Bakan B, Boivin P, Marion D. Stability of barley and malt lipid transfer protein 1 (LTP1) toward heating and reducing agents: relationships with the brewing process. J Agric Food Chem 2006; 54:3108-13. 